\title{
Pairing symmetry in the anisotropic Fermi superfluid under $p$-wave Feshbach Resonance
}

\author{
Chi-Ho Cheng and Sung-Kit Yip \\ Institute of Physics, Academia Sinica, Taipei, Taiwan
}

(Dated: July 16, 2018)

\begin{abstract}
The anisotropic Fermi superfluid of ultra-cold Fermi atoms under the $p$-wave Feshbach resonance is studied theoretically. The pairing symmetry of the ground state is determined by the strength of the atom-atom magnetic dipole interaction. It is $k_{z}$ for a strong dipole interaction; while it becomes $k_{z}-i \beta k_{y}$, up to a rotation about $\hat{z}$, for a weak one (Here $\beta<1$ is a numerical coefficient). By changing the external magnetic field or the atomic gas density, a phase transition between these two states can be driven. We discuss how the pairing symmetry of the ground state can be determined in the time-of-flight experiments.
\end{abstract}

PACS numbers: 03.75.Ss, 05.30.Fk, 34.90.+q

\section{INTRODUCTION}

The trapped Bose or Fermi atomic gases [1, 2] are usually weakly interacting since the inter-particle distances between the atoms are typically much larger than their scattering lengths. However, it has been recognized that the inter-particle interaction can in fact be tuned via Feshbach resonances [3, 4]. The interaction changes from weakly to strongly attractive by varying the external magnetic field across the Feshbach resonance. For a two-component Fermi gas, the ground state evolves from a Bardeen-Cooper-Schrieffer (BCS) superfluid with longranged (compared with inter-particle distances) Cooper pairing to a Bose-Einstein condensate (BEC) of tightly bound pairs 5, 6, 7]. This cross-over has recently been a subject of intensive theoretical [8, 9, 10, 11, 12] and experimental 13, 14, 15, 16, 17] investigations.

Most previous investigations deal with $s$-wave Feshbach resonance. Therefore, both the Cooper pairs and the bound states between two fermions have $s$-wave symmetries. Recent experiments demonstrated $p$-wave Feshbach resonances 18, 19, 20, 21]. It thus raises the possibility of $p$-wave Fermi superfluid and BEC of $p$-wave "molecules" 22, 23, 24] (see also 25]).

To begin, we first recall the well-known superfluid ${ }^{3} \mathrm{He}$ [26. ${ }^{3} \mathrm{He}$ has (nuclear) spin $1 / 2$, in general not spinpolarized and basically spatially isotropic, that is, the interaction between two atoms is dependent of the orientation of their relative distance. Back in the 60's, Anderson and Morel 27] investigated theoretically the superfluid state for this system by assuming that the pairing exists only between the same (say $\uparrow$ ) species. They showed that the ground state corresponds to Cooper pairing in the $l=1, m=1$ channel, that is, the pairing wavefunction has the symmetry of the spherical harmonic $Y_{11}(\hat{k}) \propto\left(\hat{k}_{x}+i \hat{k}_{y}\right)$ (or its spatial rotation, such as $\left.\hat{k}_{z}-i \hat{k}_{y}\right)$. This orbital symmetry is realized in the ${ }^{3} \mathrm{He}$ A-phase and is known as the "axial" state [26]. One can thus suspect that for a spin-polarized but otherwise spatially isotropic system, the pairing is again in the $Y_{11}(\hat{k})$ state. This has been confirmed in [24].

However, in the atomic gas system, as demonstrated and explained by Ticknor et al. [19] in the context of ${ }^{40} \mathrm{~K}$, the magnetic dipole interaction, which breaks rotational symmetry, may be important. (An Alkali atom has one additional electron beyond closed electronic shell(s), hence it possesses a magnetic moment mainly due to this extra electron, though the precise value of this moment depends on the hyperfine interaction and the external magnetic field). They show that, due to this magnetic dipole interaction, for magnetic field along $\hat{z}$, the $l=1$, $m=0$ resonance occurs at a higher magnetic field than the $m= \pm 1$ ones (see also 21]) For a given magnetic field, the induced effective interaction is anisotropic. In our previous paper [28] (see also [29]), we discussed theoretically the expected ground state order parameter under this circumstance. Since the interaction of the $m=0$ channel is more attractive than that of the $m= \pm 1$, in the case that the $m=0$ and $m= \pm 1$ resonances are sufficiently far apart, we showed that the pairing can only occur in the $m=0$ channel, and the ground state becomes $Y_{10}(\hat{k}) \propto k_{z}$. The system is either the BCS state with $k_{z}$ Cooper pairing or the BEC state of bosonic "molecules" in the $m=0$ channel, depending on the detuning. Fermion pairing or bosons in $m= \pm 1$ channel do not exist. The orbital symmetry of the pairing is the same as the "polar" phase in the ${ }^{3} \mathrm{He}$ literature [26].

For the case that two resonances in $m=0$ and $m= \pm 1$ channels are closed to each other, we showed that the ground state symmetry is $k_{z}-i \beta k_{y}$ up to rotation about $\hat{z}$, where $\hat{z}$ is along the magnetic field direction and $\beta<1$. This state is thus intermediate between the axial $(\beta=1)$ the the polar $(\beta=0)$ phases.

This paper provides some details of our earlier investigation [28], as well as some additional information. It is organized as follows. We first study the Cooper pairing symmetry of a weak-coupling BCS model, where the pwave pairing interaction is anisotropic. We show in that case how the analogous results cited above arise. Then we return to the slightly more involved case of Feshbach 
resonance and determine the general phase diagram for this case. We first provide the results for the case where the system can be described by an effective single channel model, i.e., purely in terms of atoms interacting with each other via a two-body instantaneous interaction. Then we study the necessary modifications to these results when this approximation is relaxed. Finally a probe of the pairing symmetry based on the time-of-flight experiment is discussed.

\section{LESSONS FROM ANISOTROPIC BCS MODEL}

In the spirit of the work of Anderson and Morel 27], we first consider a weak-coupling BCS model of a Fermi system with one spin species under the interaction $-V_{\vec{k}-\vec{k}^{\prime}}$. (We shall however study the case where this $V$ is anisotropic, see below). The Hamiltonian of our system is $H=H_{f}+H_{V}$, where

$$
\begin{gathered}
H_{f}=\sum_{\vec{k}}\left(\epsilon_{k}-\mu\right) a_{\vec{k}}^{\dagger} a_{\vec{k}} \\
H_{V}=-\sum_{\vec{k}, \vec{k}^{\prime}, \vec{q}} V_{\vec{k}-\vec{k}^{\prime}} a_{\vec{q}+\vec{k}^{\prime}}^{\dagger} a_{\vec{q}-\vec{k}^{\prime}}^{\dagger} a_{\vec{q}-\vec{k}} a_{\vec{q}+\vec{k}}
\end{gathered}
$$

Here $\epsilon_{k}=\hbar^{2} k^{2} / 2 M$, and $\mu$ is the chemical potential, and $a_{\vec{k}}$ is the annihilation operator for a Fermion with wavevector $\vec{k}$. Standard BCS theory proposes the following trial ground state wavefunction

$$
|G\rangle=\prod_{k}\left(u_{\vec{k}}+v_{\vec{k}} a_{\vec{k}}^{\dagger} a_{-\vec{k}}^{\dagger}\right)|0\rangle
$$

with normalization condition $\left|u_{\vec{k}}\right|^{2}+\left|v_{\vec{k}}\right|^{2}=1$. Minimizing the ground state energy $\langle G|H| G\rangle$ with respect to $u_{\vec{k}}$ and $v_{\vec{k}}$ gives the excitation spectrum

$$
E_{\vec{k}}=\sqrt{\left(\epsilon_{k}-\mu\right)^{2}+\left|\Delta_{\vec{k}}\right|^{2}}
$$

where the energy gap $\Delta_{\vec{k}}$ satisfies

$$
\Delta_{\vec{k}}=\sum_{\vec{k}^{\prime}} V_{\vec{k}-\vec{k}^{\prime}} \frac{\Delta_{\vec{k}^{\prime}}}{2 E_{\vec{k}^{\prime}}}
$$

In case the interaction is $p$-wave, only the $l=1$ component survive in $V_{\vec{k}-\vec{k}^{\prime}}$. We write

$$
V_{\vec{k}-\vec{k}^{\prime}}=2 \pi \sum_{m=-1}^{m=1} V_{m}\left(k, k^{\prime}\right) Y_{1 m}(\hat{k}) Y_{1 m}^{*}\left(\hat{k}^{\prime}\right)
$$

We shall study the situation where $V_{1}=V_{-1} \leq V_{0}$. We also decompose the energy gap in spherical harmonics of $l=1$ partial waves,

$$
\begin{aligned}
\Delta_{\vec{k}} & =\Delta f(\hat{k}) \\
& =\Delta \sum_{m=-1}^{m=1} f_{m} Y_{1 m}(\hat{k})
\end{aligned}
$$

with normalization $\sum_{m}\left|f_{m}\right|^{2}=1$. Substituting Eqs.(6)(7) into Eq.(5), we have

$f(\hat{k})=\pi \sum_{\vec{k}^{\prime}} \sum_{m} V_{m}\left(k, k^{\prime}\right) Y_{1 m}(\hat{k}) \frac{Y_{1 m}^{*}\left(\hat{k}^{\prime}\right) f\left(\hat{k}^{\prime}\right)}{\left[\left(\epsilon_{k^{\prime}}-\mu\right)^{2}+\Delta^{2}\left|f\left(\hat{k}^{\prime}\right)\right|^{2}\right]^{\frac{1}{2}}}$

Suppose further that $V_{m}\left(k, k^{\prime}\right)=V_{m}$ is independent of $k$ and $k^{\prime}$, Eq. (8) becomes

$$
f_{m}=\pi V_{m} \sum_{\vec{k}} \frac{Y_{1 m}^{*}(\hat{k}) f(\hat{k})}{\left[\left(\epsilon_{k}-\mu\right)^{2}+\Delta^{2}|f(\hat{k})|^{2}\right]^{\frac{1}{2}}}
$$

We perform the integration in $\vec{k}$ in the following way,

$$
\sum_{\vec{k}} \rightarrow \rho_{0} \int_{-\Lambda}^{\Lambda} d \epsilon \int d \Omega
$$

where $\rho_{0}$ is the density of state around the Fermi surface, $\Lambda$ is the energy cutoff, and $d \Omega$ is solid angle. Then we get totally 3 nonlinear equations,

$f_{m}=2 \pi \rho_{0} V_{m}\left[f_{m} \log \left(\frac{2 \Lambda}{\Delta}\right)-\int d \Omega Y_{1 m}^{*}(\hat{k}) f(\hat{k}) \log |f(\hat{k})|\right]$

for $m=-1,0,1$.

We would like to find the solution to Eq. (11) with the anisotropy characterized by the ratio $V_{1} / V_{0}$. Particular solutions to Eq. (11) can be easily found at two limits. When $V_{1} / V_{0}=0, \Delta_{\vec{k}} \propto Y_{10}(\hat{k}) \propto k_{z}$ because no other pairing can be possible. At the other limit, $V_{1} / V_{0}=1$, the system is again isotropic the ground state pairing is $\Delta_{\vec{k}} \propto Y_{11}(\hat{k})$, or its rotations. In terms of the language by $f_{m}$ 's, for $V_{1} / V_{0}=0, f_{0}=1, f_{1}=f_{-1}=0$ (up to a gauge transformation). For $V_{1} / V_{0}=1$, a particular solution is $f_{0}=1 / \sqrt{2}, f_{1}=f_{-1}=1 / 2$ such that

$$
\begin{aligned}
\Delta_{\vec{k}} & \propto \frac{1}{\sqrt{2}} Y_{10}(\hat{k})+\frac{1}{2}\left(Y_{11}(\hat{k})+Y_{1,-1}(\hat{k})\right) \\
& \propto k_{z}-i k_{y}
\end{aligned}
$$

Note that it does not contradict with the pairing $Y_{11}(\hat{k}) \propto$ $k_{x}+i k_{y}$ since these states are degenerate in an isotropic limit $V_{1}=V_{-1}=V_{0}$. With gradually tuning the parameter $0<V_{1} / V_{0}<1$, we will show below that $\Delta(\hat{k}) \propto k_{z}-i \beta k_{y}$ up to a $z$-axis rotation with $\beta<1$.

Before solving the gap equation Eq. (11) for the general intermediate values of the pairing $0<V_{1} / V_{0}<1$, it is 
helpful to first identify the critical $V_{1}^{*} / V_{0}$ such that $f_{1}$ and $f_{-1}$ start to deviate from zero (i.e., when the pairing starts to deviate from $Y_{10}$ ). Let us take the gauge where $f_{0}$ is real. Linearizing Eq. (11) in $f_{1}$ and $f_{-1}$ around the point $\left(f_{0}, f_{1}, f_{-1}\right)=(1,0,0)$, we have

$$
\frac{1}{2 \pi \rho_{0} V_{1}^{*}}\left(\begin{array}{c}
f_{1} \\
f_{-1}^{*}
\end{array}\right)=\left(\begin{array}{cc}
A_{1} & A_{2} \\
A_{2} & A_{1}
\end{array}\right)\left(\begin{array}{c}
f_{1} \\
f_{-1}^{*}
\end{array}\right)
$$

where $A_{1}=\log (2 \Lambda / \Delta)-\int d \Omega\left|Y_{11}\right|^{2} \log \left|Y_{10}\right|-1 / 2, A_{2}=$ $1 / 2$. Thus when we increase $V_{1}$, the first non-trivial solution for $f_{1}$ and $f_{-1}$ is obtained when $\left(f_{1}, f_{-1}^{*}\right)$ gives the largest eigenvalue of the matrix. It can be easily seen that this corresponds to the solution $f_{1}=f_{-1}^{*}$, with eigenvalue $A_{1}+A_{2}$. The critical value $V_{1}^{*}$ thus satisfies

$$
\frac{1}{2 \pi \rho_{0} V_{1}^{*}}=\left[\log \left(\frac{2 \Lambda}{\Delta}\right)-\int d \Omega\left|Y_{11}\right|^{2} \log \left|Y_{10}\right|\right]
$$

On the other hand, at this point (from Eq. (11) with $m=0)$,

$$
\frac{1}{2 \pi \rho_{0} V_{0}}=\left[\log \left(\frac{2 \Lambda}{\Delta}\right)-\int d \Omega\left|Y_{10}\right|^{2} \log \left|Y_{10}\right|\right]
$$

Eliminating $\log (2 \Lambda / \Delta)$ in Eqs.(14)-(15) gives

$$
V_{1}^{*} / V_{0}=\frac{1}{1+2 \pi \rho_{0} V_{0}}
$$

For $V_{1}^{*} / V_{0}<V_{1} / V_{0}<1, f_{ \pm 1}$ becomes finite. Provided no additional phase transitions occur, we expect then the relation $f_{1}=f_{-1}^{*}$ remains valid. Furthermore, our system is rotationally invariant around $\hat{z}$. Under this rotation, $f_{1} \rightarrow f_{1} e^{i \phi}$ but $f_{-1} \rightarrow f_{-1} e^{-i \phi}$ where $\phi$ is the rotational angle. By suitable choice of $\phi$ we can thus make both $f_{1}$ and $f_{-1}=f_{1}^{*}$ real and positive. We shall confine ourselves to this case without loss of generality. Under this choice the order parameter has the form $k_{z}-$ $i \beta k_{y}$.

Hence, when $V_{1}^{*} / V_{0}<V_{1} / V_{0}<1$, we search for the solution $f_{0}>0, f_{1}=f_{-1}>0$ under the normalization condition $f_{0}^{2}+2 f_{1}^{2}=1$. The solution $f_{0}$ (where $\left.f_{1}=f_{-1}=\sqrt{\left(1-f_{0}^{2}\right) / 2}\right)$ for different $V_{1} / V_{0}$ and $\rho_{0} V_{0}$ is presented in Fig प For given $\rho_{0} V_{0}$, the pairing is $k_{z}$ $\left(f_{0}=0\right)$ at small $V_{1} / V_{0}$ until the latter exceeds the critical $V_{1}^{*} / V_{0}$. Beyond this critical $V_{1}^{*} / V_{0}, f_{0}$ decreases gradually with increasing $V_{1} / V_{0}$. At $V_{1} / V_{0}=1, f_{0}=1 / \sqrt{2}$ for all $\rho_{0} V_{0}$ 's meaning that the pairing becomes $k_{z}-i k_{y}$ (corresponding to $\beta=1$ ).

\section{P-WAVE FESHBACH PAIRING}

We return to the case of p-wave Feshbach resonance. We begin with the total Hamiltonian $H=H_{f}+H_{b}+H_{\alpha}$ where again $H_{f}$ is for the free fermions,

$$
H_{f}=\sum_{\vec{k}}\left(\epsilon_{k}-\mu\right) a_{\vec{k}}^{\dagger} a_{\vec{k}} .
$$

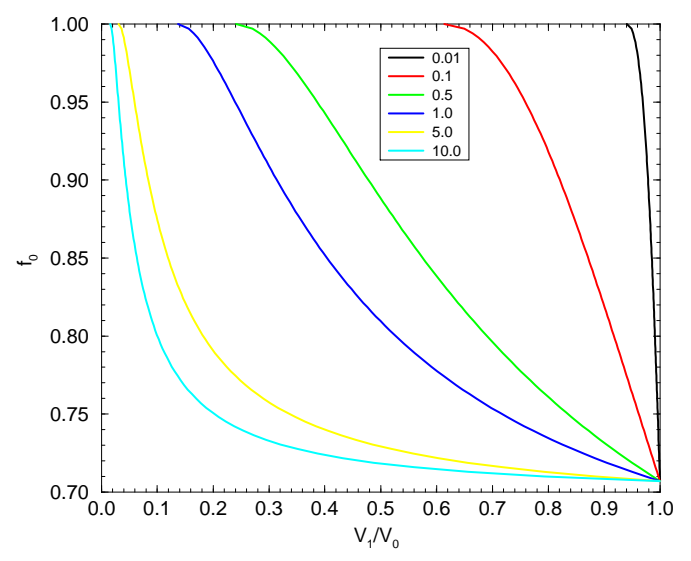

FIG. 1: $f_{0}$ as a function of $V_{1} / V_{0}$ for $\rho_{0} V_{0}$ 's given in the legend.

$H_{b}$ is for the molecules in the "closed" channels, with $b_{\vec{q}, m}$ the annihilation operator for a Boson with angular momentum $l=1$ and $z$-axis projection $m$ with momentum $\vec{q}$,

$$
H_{b}=\sum_{\vec{q}}\left(\frac{\epsilon_{q}}{2}+\delta_{m}-2 \mu\right) b_{\vec{q}, m}^{\dagger} b_{\vec{q}, m}
$$

Here $\delta_{m}$ 's are the (bare) detuning of the $l=1, m$ resonance. $H_{\alpha}$ represents the Feshbach coupling, which is a generalization of the ones already commonly employed [8, 30] for $s$-wave Feshbach resonances to the case of several $l=1, m=0, \pm 1$ closed channels [23, 24]:

$$
H_{\alpha}=\sum_{m, \vec{q}, \vec{k}} \varphi_{m}^{*}(\vec{k}) b_{\vec{q}, m}^{\dagger} a_{-\vec{k}+\vec{q} / 2} a_{\vec{k}+\vec{q} / 2}+\text { h.c. }
$$

where $\varphi_{m}(\vec{k})=-\frac{i \sqrt{4 \pi}}{L^{3 / 2}} k Y_{1 m}(\hat{k}) \alpha_{m}$. The factor $Y_{1 m}(\hat{k})$ reflects the symmetry of the $l, m$ bound state and the linear factor in $k$ arises from the small momentum approximation for the coupling. $\alpha_{m}$ is the corresponding coupling constant, it has dimension $[E][L]^{5 / 2}$. More precisely $\alpha_{m}$ can be an analytic function of $k$. For low energy (density) phenomena, we shall however only need its value $\alpha_{m} \equiv \alpha_{m}(k=0)$.

We shall apply mean-field theory to the Hamiltonian $H$. In this approximation, we discard all terms that involve finite momentum Bosons $b_{\vec{q}, m}$ with $\vec{q} \neq 0$, and regard $b_{\vec{q}=0, m}$ as c-numbers. It is then convenient to introduce

$$
\begin{aligned}
D_{m} & =-\frac{i \sqrt{4 \pi}}{L^{\frac{3}{2}}} \alpha_{m} b_{0, m} \\
\Delta_{\vec{k}} & =\sum_{m} D_{m} k Y_{1 m}(\hat{k})
\end{aligned}
$$

Hence the mean-field Hamiltonian for the Fermions be- 
comes

$$
H^{\mathrm{mf}}=\sum_{\vec{k}}\left(\epsilon_{k}-\mu\right) a_{\vec{k}}^{\dagger} a_{\vec{k}}+\left(\Delta_{\vec{k}}^{*} a_{-\vec{k}} a_{\vec{k}}+\text { h.c. }\right)
$$

This can be solved via the familiar Bogoliubov transformation, which gives

$$
\left\langle a_{-\vec{k}} a_{\vec{k}}\right\rangle=-\frac{\Delta_{\vec{k}}}{2\left[\left(\epsilon_{k}-\mu\right)^{2}+\left|\Delta_{\vec{k}}\right|^{2}\right]^{\frac{1}{2}}}
$$

Minimization with respect to the c-numbers $b_{\vec{q}=0, m}$ give

$$
b_{0, m}\left(\delta_{m}-2 \mu\right)=-\sum_{\vec{k}} \varphi_{m}^{*}(\vec{k})\left\langle a_{-\vec{k}} a_{\vec{k}}\right\rangle
$$

which can be written as,

$$
\left(\delta_{m}-2 \mu\right) D_{m}=\frac{2 \pi}{L^{3}} \sum_{\vec{k}} \frac{\left|\alpha_{m}\right|^{2} k Y_{1 m}^{*}(\hat{k}) \Delta_{\vec{k}}}{\left[\left(\epsilon_{k}-\mu\right)^{2}+\left|\Delta_{\vec{k}}\right|^{2}\right]^{\frac{1}{2}}}
$$

The R.H.S. of this equation is formally divergent (This divergence is only formal: the sum may actually converge if the $k$ dependences of $\alpha_{m}(k)$ are included) Nevertheless, it can be "renormalized" by re-expressing this equation in terms of parameters that enter the two-body scattering amplitude (c.f. 24 and also the theoretical references in the s-wave case $[5,9,10,11,12$.) Let us then consider the scattering of two particles in vacuum. We denote $f_{\vec{k}}\left(\vec{k}^{\prime}\right)$ as the scattering amplitude for two incident particles with relative momentum $\vec{k}$ and out-going momentum $\vec{k}^{\prime}$. We can define $f_{m}(k)$ (do not confuse this $f_{m}(k)$ with those in Sec II) via

$$
f_{\vec{k}}\left(\vec{k}^{\prime}\right)=\sum_{m} f_{m}(k)(4 \pi) Y_{1 m}\left(\hat{k}^{\prime}\right) Y_{1 m}^{*}(\hat{k})
$$

This equation is the generalization of the corresponding one in scattering theory to the case of anisotropic interaction (but still with rotational invariance around $\hat{z}$ ). It reduces to those in standard quantum mechanics textbooks [31] if $f_{m}(k)$ is independent of $m . f_{\vec{k}}\left(\vec{k}^{\prime}\right)$ is related to the $T$-matrix by

$$
f_{\vec{k}}\left(\vec{k}^{\prime}\right)=-\frac{M L^{3}}{4 \pi \hbar^{2}} T_{\vec{k}^{\prime}, \vec{k}}\left(E=2 \epsilon_{k}+i 0^{+}\right)
$$

The $T$-matrix can be evaluated in the standard way:

$$
\begin{aligned}
T_{\vec{k}^{\prime}, \vec{k}}(E)= & \sum_{m}\left[\varphi_{m}\left(\vec{k}^{\prime}\right) \mathcal{G}_{b_{0}}^{(m)}(E) \varphi_{m}^{*}(\vec{k})\right. \\
& \left.+\varphi_{m}\left(\vec{k}^{\prime}\right) \mathcal{G}_{b_{0}}^{(m)}(E) \Pi^{(m m)}(E) \mathcal{G}_{b_{0}}^{(m)}(E) \varphi_{m}^{*}(\vec{k})+\cdots\right] \\
= & \sum_{m} \frac{\varphi_{m}^{*}\left(\vec{k}^{\prime}\right) \varphi_{m}(\vec{k})}{E-\delta_{m}-\Pi^{(m)}(E)}
\end{aligned}
$$

where

$$
\begin{aligned}
\Pi^{\left(m m^{\prime}\right)}(E) & =\sum_{\vec{p}, \omega} \varphi_{m}^{*}(\vec{p}) \mathcal{G}_{f}(\vec{p}, \omega) \mathcal{G}_{f}(-\vec{p}, E-\omega) \varphi_{m^{\prime}}(\vec{p}) \\
& =\sum_{\vec{p}} \frac{\varphi_{m}^{*}(\vec{p}) \varphi_{m^{\prime}}(\vec{p})}{E-2 \epsilon_{p}}
\end{aligned}
$$
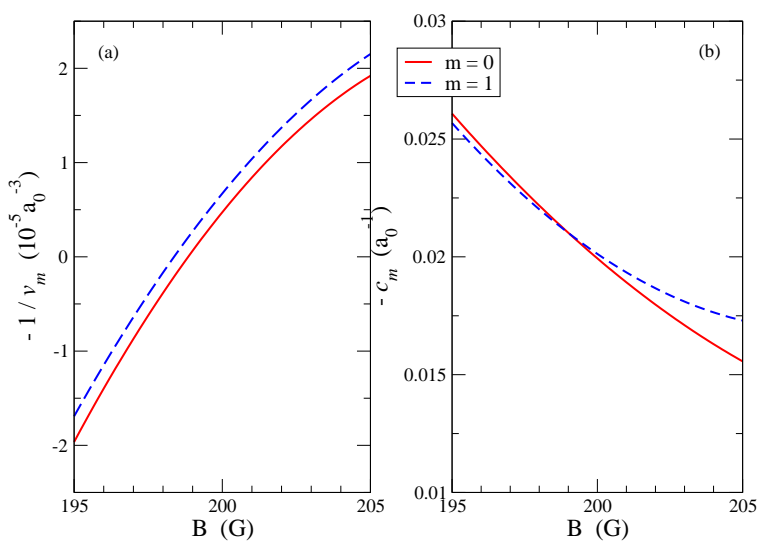

FIG. 2: Plot of the fit to the experimental results of Ref. 19] for $-1 / v_{m}$ and $-c_{m}$ for ${ }^{40} K$ atoms in the $\left|f, m_{f}\right\rangle=$ $|9 / 2,-7 / 2\rangle$ hyperfine state. $v_{1}=v_{-1}$ and $c_{1}=c_{-1} .-1 / v_{m}$ 's vanish at the resonant fields $B_{m}^{*}$, with $B_{0}^{*}>B_{ \pm 1}^{*}$.

$\mathcal{G}_{f}(\vec{p}, \omega)$ is the Greens function for free Fermions at wavevector $\vec{p}$ ( $p$ has dimension of $[L]^{-1}$ ), frequency $\omega$, and $\mathcal{G}_{b_{0}}^{(m)}(E)$ is the Greens functions of free $m$-Boson at zero momentum $\vec{q}=0$ and energy $E$. Here we have already made use of a simplification due to the fact that $\Pi^{\left(m m^{\prime}\right)}$ vanishes for $m \neq m^{\prime}$ (see Eq. (29)), hence there are no cross-terms between different $m$ 's in Eq. (28).

Substituting Eqs.(28)-(29) into Eq.(27), and using the definition (26), we get

$$
-\frac{M}{4 \pi \hbar^{2}} \frac{k^{2}}{f_{m}(k)}=\frac{2 \epsilon_{k}-\delta_{m}}{\left|\alpha_{m}\right|^{2}}-\frac{1}{L^{3}} \sum_{\vec{p}} \frac{p^{2}}{2 \epsilon_{k}-2 \epsilon_{p}+i 0^{+}}
$$

The sum over $\vec{p}$ in the R.H.S. formally diverges. However, we are interested only on its dependence on $k$ at small $k$ 's. We thus expand the R.H.S. in powers of $k$ :

$$
\begin{aligned}
& \frac{2 \epsilon_{k}-\delta_{m}}{\left|\alpha_{m}\right|^{2}}-\frac{1}{L^{3}} \sum_{\vec{p}} \frac{p^{2}}{2 \epsilon_{k}-2 \epsilon_{p}+i 0^{+}} \\
= & -\left(\frac{\delta_{m}}{\left|\alpha_{m}\right|^{2}}-\frac{M}{\hbar^{2}} \frac{1}{L^{3}} \sum_{\vec{p}} 1\right) \\
& +\left(\frac{\hbar^{2}}{M\left|\alpha_{m}\right|^{2}}+\frac{M}{\hbar^{2}} \frac{1}{L^{3}} \sum_{\vec{p}} \frac{1}{p^{2}}\right) k^{2}-i \frac{M}{4 \pi \hbar^{2}} k^{3}
\end{aligned}
$$

In the low-energy limit, Eq.(30)-(31) should reduce to (28the general result for the low-energy scattering between a pair of particles. Using the same notation adopted in Ref. [19], the scattering amplitude is parametrized as

$$
f_{m}(k)=\frac{k^{2}}{-v_{m}^{-1}+c_{m} k^{2}-i k^{3}}
$$

where the dimensions of $v_{m}$ and $c_{m}$ are $[\mathrm{L}]^{3}$ and $[\mathrm{L}]^{-1}$, respectively. The magnetic field dependent parameters $v_{m}$, 
$c_{m}$ are in principle available experimentally. An example is as shown in Fig 2 Our renormalization scheme is to absorb the bare parameters $\delta_{m}$ and $\alpha_{m}$ into the physical (renormalized) parameters $v_{m}, c_{m}$ by identifying the equivalence between Eqs.(30)-(31) and Eq.(32). Hence

$$
\begin{aligned}
-\frac{1}{v_{m}} & =\frac{4 \pi \hbar^{2}}{M}\left(\frac{\delta_{m}}{\left|\alpha_{m}\right|^{2}}-\frac{M}{\hbar^{2}} \frac{1}{L^{3}} \sum_{\vec{p}} 1\right) \\
c_{m} & =-\frac{4 \pi \hbar^{2}}{M}\left(\frac{\hbar^{2}}{M\left|\alpha_{m}\right|^{2}}+\frac{M}{\hbar^{2}} \frac{1}{L^{3}} \sum_{\vec{p}} \frac{1}{p^{2}}\right)
\end{aligned}
$$

In Eq. (33) and (34), if the $p$ dependence of $\alpha_{m}$ 's had been included, the sums involving $\vec{p}$ should have the extra factors $\left|\alpha_{m}(p)\right|^{2} /\left|\alpha_{m}(0)\right|^{2}$. When treating the many-body problem, as we shall see below, the only "divergent" sums are again $\sum_{\vec{p}}\left|\alpha_{m}(p)\right|^{2} /\left|\alpha_{m}(0)\right|^{2}$ and $\sum_{\vec{p}}\left|\alpha_{m}(p)\right|^{2} /\left|\alpha_{m}(0)\right|^{2} p^{2}$. We shall apply Eq. (33) and (34) to eliminate these sums in favor of $v_{m}$ and $c_{m}$. After this procedure, low energy phenomena (involving only small $p$ 's) can be parameterized entirely by $v_{m}, c_{m}$ and $\alpha_{m}(0)$. To simplify our presentation, we shall not include explicitly the factors $\left|\alpha_{m}(p)\right|^{2} /\left|\alpha_{m}(0)\right|^{2}$ in our calculations below.

Returning to Eq.25), we rewrite its R.H.S as

$$
\begin{aligned}
& \frac{2 \pi}{L^{3}} \sum_{\vec{k}} \frac{\left|\alpha_{m}\right|^{2} k Y_{1 m}^{*}(\hat{k}) \Delta_{\vec{k}}}{\left[\left(\epsilon_{k}-\mu\right)^{2}+\left|\Delta_{\vec{k}}\right|^{2}\right]^{\frac{1}{2}}} \\
= & \frac{2 \pi\left|\alpha_{m}\right|^{2}}{L^{3}} \sum_{\vec{k}, m^{\prime}} \frac{D_{m^{\prime}} Y_{1 m}^{*}(\hat{k}) Y_{1 m^{\prime}}(\hat{k}) k^{2}}{\left[\left(\epsilon_{k}-\mu\right)^{2}+\left|\Delta_{\vec{k}}\right|^{2}\right]^{\frac{1}{2}}} \\
= & \frac{2 \pi\left|\alpha_{m}\right|^{2}}{L^{3}} \sum_{\vec{k}, m^{\prime}} D_{m^{\prime}} Y_{1 m}^{*}(\hat{k}) Y_{1 m^{\prime}}(\hat{k}) \\
& \times\left\{h(\vec{k})+\frac{k^{2}}{\epsilon_{k}}\left(1+\frac{\mu}{\epsilon_{k}}-\frac{\left|\Delta_{\vec{k}}\right|^{2}}{2 \epsilon_{k}^{2}}\right)\right\}
\end{aligned}
$$

where

$$
h(\vec{k}) \equiv \frac{k^{2}}{\left[\left(\epsilon_{k}-\mu\right)^{2}+\left|\Delta_{\vec{k}}\right|^{2}\right]^{1 / 2}}-\frac{k^{2}}{\epsilon_{k}}\left(1+\frac{\mu}{\epsilon_{k}}-\frac{\left|\Delta_{\vec{k}}\right|^{2}}{2 \epsilon_{k}^{2}}\right)
$$

The sum involving $h(\vec{k})$ in Eq. (35) is convergent. For the rest of the terms, their divergences are the same as in either Eqs. (33) and (34). These sums can thus be expressed in terms of the physical parameters $v_{m}$ and $c_{m}$. Using Eq.(35), the "gap equation" Eq.(25) can therefore be rewritten as

$$
\begin{aligned}
& \frac{M}{4 \pi} D_{0}\left(-\frac{1}{v_{0}}+\frac{2 M c_{0} \mu}{\hbar^{2}}\right)-\frac{3 M}{10 \pi}\left(\frac{M^{2} c_{0}}{4 \pi \hbar^{4}}+\frac{1}{\left|\alpha_{0}\right|^{2}}\right)\left[D_{0}\left(2\left|D_{1}\right|^{2}+3\left|D_{0}\right|^{2}+2\left|D_{-1}\right|^{2}\right)-2 D_{0}^{*} D_{1} D_{-1}\right] \\
& =\frac{2 \pi}{L^{3}} \sum_{\vec{k}, m} D_{m} Y_{10}^{*}(\hat{k}) Y_{1 m}(\hat{k}) h(\vec{k}) \\
& \frac{M}{4 \pi} D_{1}\left(-\frac{1}{v_{1}}+\frac{2 M c_{1} \mu}{\hbar^{2}}\right)-\frac{3 M}{10 \pi}\left(\frac{M^{2} c_{1}}{4 \pi \hbar^{4}}+\frac{1}{\left|\alpha_{1}\right|^{2}}\right)\left[D_{1}\left(3\left|D_{1}\right|^{2}+2\left|D_{0}\right|^{2}+6\left|D_{-1}\right|^{2}\right)-D_{-1}^{*} D_{0}^{2}\right] \\
& =\frac{2 \pi}{L^{3}} \sum_{\vec{k}, m} D_{m} Y_{11}^{*}(\hat{k}) Y_{1 m}(\hat{k}) h(\vec{k})
\end{aligned}
$$

and a corresponding equation with $m=1 \leftrightarrow m=-1$. Eq.(37)-(38) are to be solved under the constraint from 
the number equation

$$
n=\frac{1}{L^{3}}\left(\sum_{\vec{k}}\left\langle a_{\vec{k}}^{\dagger} a_{\vec{k}}\right\rangle+2 \sum_{m}\left|b_{0, m}\right|^{2}\right)
$$

The first term is from open-channel atoms whereas the second term is from the closed-channel molecules. From BCS theory,

$$
\left\langle a_{\vec{k}}^{\dagger} a_{\vec{k}}\right\rangle=\frac{1}{2}\left(1-\frac{\epsilon_{k}-\mu}{\left[\left(\epsilon_{k}-\mu\right)^{2}+\left|\Delta_{\vec{k}}\right|^{2}\right]^{\frac{1}{2}}}\right)
$$

The first term in Eq. (39) is thus again formally divergent: $\left\langle a_{\vec{k}}^{\dagger} a_{\vec{k}}\right\rangle \sim \frac{\left|\Delta_{\vec{k}}\right|^{2}}{4 \epsilon_{k}^{2}}$ at large $k$. However, this sum it can be treated in analogous manner as Eq. (35) by employing again Eq. (34). The result is

$$
\begin{aligned}
n= & \frac{1}{2 L^{3}} \sum_{\vec{k}}\left(1-\frac{\epsilon_{k}-\mu}{\left[\left(\epsilon_{k}-\mu\right)^{2}+\left|\Delta_{\vec{k}}\right|^{2}\right]^{\frac{1}{2}}}-\frac{\left|\Delta_{\vec{k}}\right|^{2}}{2 \epsilon_{k}^{2}}\right) \\
& -\frac{1}{4 \pi} \sum_{m}\left(\frac{M^{2} c_{m}}{4 \pi \hbar^{4}}+\frac{1}{\left|\alpha_{m}\right|^{2}}\right)\left|D_{m}\right|^{2}+\frac{1}{2 \pi} \sum_{m} \frac{\left|D_{m}\right|^{2}}{\left|\alpha_{m}\right|^{2}}
\end{aligned}
$$

Eqs.(37), (38), and (40) are our principal equations, with parameters characterizing the Feshbach resonances entirely in terms of $v_{m}, c_{m}$ and $1 /\left|\alpha_{m}\right|^{2}$. These equations determine the order parameters $D_{m}$ and chemical potential $\mu$ for given density $n$ and "interaction parameters" $v_{m}, c_{m}$ and $1 /\left|\alpha_{m}\right|^{2}$.

\section{BEC-BCS CROSSOVER AND QUANTUM PHASE TRANSITIONS}

Generally with Feshbach resonance for the sub-channel $m, 1 / v_{m}$ is field dependent, vanishing at the resonant field $B_{m}^{*}$. In contrast, $c_{m}$ has a definite sign (c.f. Fig. 21). For the ease of discussions, we shall assume that $c_{m}<0$ and field independent, $-1 / v_{m}$ is an increasing function of field $-1 / v_{m}>(<) 0$ for $B>(<) B_{m}^{*}$, as in the case of ${ }^{40} \mathrm{~K}$. (This corresponds to the case where $\delta_{m}$ is an increasing function of field and $\alpha_{m}$ weakly field dependent, c.f. Eqs.(33) and (34)). For $B<B_{m}^{*}$, a bound state appears. The energy of this bound state is given by $-\epsilon_{b, m}=-\hbar^{2} \kappa_{m}^{2} / M$ with $k=i \kappa_{m}$ being a pole for $f_{m}(k)$, that is, $-1 / v_{m}-c_{m} \kappa_{m}^{2}-\kappa_{m}^{3}=0$. For given $m$ and small detuning below that resonance, $\kappa_{m}$ is small and is given by $\kappa_{m}^{2}=1 /\left[\left(-c_{m}\right)\left(v_{m}\right)\right]$. Since $1 / v_{m}$ should be roughly linear in $B$ near the resonance, $\epsilon_{b, m}$ increases linearly with $\left(B_{m}^{*}-B\right)$ (in contrast to $s$-wave, where it is quadratic; see also 24]) These results for small detuning apply provided $\kappa_{m}<<\left(-c_{m}\right)$, or equivalently $1 / v_{m} \ll\left(-c_{m}\right)^{3}$. We shall always confine ourselves to this regime for negative detunings (even when we write $\left.B \ll B_{m}^{*}\right)$. For larger negative detunings we need to take into account higher order terms in $k^{2}$ in denominator of $f_{m}(k)$.

Moreover, as explained in Ref. [19], due to the dipole interaction, $B_{0}^{*}>B_{1}^{*}=B_{-1}^{*}$, as can be seen again in Fig 2 Thus in the field range of interest, $-1 / v_{1}=-1 / v_{-1}>$ $-1 / v_{0}$. We can say that, at a given field, the effective interaction between the Fermions is less attractive for relative angular momentum projections $m= \pm 1$ than $m=0$.

Since the interaction is less attractive for angular momentum projections $m= \pm 1$, for sufficiently large difference between $-1 / v_{0}$ and $-1 / v_{ \pm 1}$ we expect (and verify below) that the pairing is entirely in the $m=0$ partial wave. We thus first begin our analysis by assuming that only $D_{0}$ is non-vanishing.

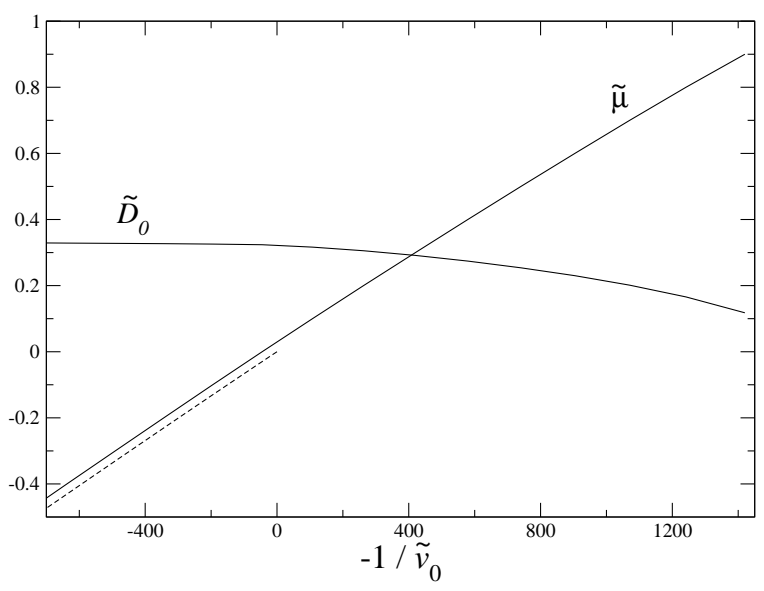

FIG. 3: The dimensionless parameters $\tilde{D}_{0}$ and $\tilde{\mu}$ as functions of $-1 / \tilde{v}_{0} . \quad \tilde{c}_{0}=\tilde{c}_{1}=-100$ and $\tilde{D}_{ \pm 1}=0$ in this case. The dashed line represents $-\epsilon_{b} / 2 \epsilon_{F}$.

For simplicity, we shall first drop the terms with explicit $1 /\left|\alpha_{m}\right|^{2}$ factors. If this is valid, then our equations (37), (38), (40) are same as a corresponding model consisting only of Fermions interacting among each other with an interaction such that the corresponding twobody scattering amplitude parameters are given by the same $c_{m}$ and $v_{m}$. In analogy with the s-wave literature, we shall call this the single-channel approximation (note there are still three $(m=-1,0,1)$ Feshbach resonances). The effect from finite $1 /\left|\alpha_{m}\right|^{2}$ will be discussed at the end of this Section.

With these simplifications, Eq. (37) and (40) become

$$
\frac{M}{4 \pi}\left(-\frac{1}{v_{0}}+\frac{2 M c_{0} \mu}{\hbar^{2}}\right)+\frac{9 M}{40 \pi^{2} \hbar^{4}}\left(-c_{0}\right)\left|D_{0}\right|^{2}=\frac{2 \pi}{L^{3}} \sum_{\vec{k}, m}\left|Y_{10}(\hat{k})\right|^{2} h(\vec{k})
$$


and

$$
\begin{aligned}
n= & \frac{1}{2 L^{3}} \sum_{\vec{k}}\left(1-\frac{\epsilon_{k}-\mu}{\left[\left(\epsilon_{k}-\mu\right)^{2}+\left|\Delta_{\vec{k}}\right|^{2}\right]^{\frac{1}{2}}}-\frac{\left|\Delta_{\vec{k}}\right|^{2}}{2 \epsilon_{k}^{2}}\right) \\
& +\frac{M^{2}}{(4 \pi)^{2} \hbar^{4}}\left(-c_{0}\right)\left|D_{0}\right|^{2}
\end{aligned}
$$

respectively. Eqs.(37) and (40) can be solved simultaneously similar to the $s$-wave case. By gauge invariance, we shall choose $D_{0}$ to be real without loss of generality. It is convenient to express the results in dimensionless form. We thus define $\tilde{\mu} \equiv \mu / \epsilon_{\mathrm{F}}, \tilde{D}_{m} \equiv D_{m} / \hbar v_{\mathrm{F}}$, $\tilde{c}_{m} \equiv n^{-1 / 3} c_{m}$, and $\tilde{v}_{m} \equiv n v_{m}$ where $\epsilon_{\mathrm{F}} \equiv \hbar^{2} k_{\mathrm{F}}^{2} / 2 M$, $v_{\mathrm{F}} \equiv \hbar k_{\mathrm{F}} / M$, and $k_{\mathrm{F}}^{3} \equiv 6 \pi^{2} n$. The results are as shown in Fig. 3 (for the case $\tilde{c}_{0}=\tilde{c}_{1}=-100$, see below for the reason of this choice).

In the BCS regime, corresponding to "large" external magnetic field $B \gg B_{0}^{*}$ thus $-\tilde{v}_{0}^{-1} \rightarrow \infty$, we have $\tilde{\mu} \simeq 1$, and $\tilde{D}_{0} \simeq 0$. We can ignore the term $\propto\left|D_{0}\right|^{2}$ on the left hand side of Eq. (41). This equation then reduces to the corresponding one in Sec II with the effective pairing interaction $V_{0}$ in the $m=0$ channel there proportional to $\left(-1 / v_{0}+2 M c_{0} \mu / \hbar^{2}\right)^{-1}=\left(-1 / v_{0}+c_{0} k_{F}^{2}\right)^{-1}$. This combination follows from the factor $\left(\delta_{0}-2 \mu\right)$ on the left hand side of Eq. (25).

In the BEC regime, corresponding to $B \ll B_{0}^{*}$ or $-\tilde{v}_{0}^{-1} \rightarrow-\infty, \mu \simeq-\epsilon_{b, 0} / 2$, and $\tilde{D}_{0}$ approaches to a constant. In Eq. (41) we can ignore all terms explicit in $\Delta_{\vec{k}}$ or $D_{0}$. Writing $\kappa=(2 M|\mu|)^{1 / 2}$ and performing the sum over $\vec{k}$, we find that $\kappa$ obeys $-1 / v_{0}-c_{0} \kappa^{2}=\kappa^{3}$. Comparing this with the equation for the two body bound state, we find $|\mu|=\epsilon_{b 0} / 2$ as claimed. In Eq. (42), we can expand the square-root $\left[\left(\epsilon_{k}-\mu\right)+\left|\Delta_{\vec{k}}\right|^{2}\right]^{-1 / 2} \approx$ $\left(\epsilon_{k}+|\mu|\right)-\left|\Delta_{\vec{k}}\right|^{2} / 2\left(\epsilon_{k}+|\mu|\right)$. We then get

$$
n=\frac{M^{2}}{(4 \pi)^{2} \hbar^{4}}\left|D_{0}\right|^{2}\left[\left(-c_{0}\right)-\frac{3}{2} \kappa\right]
$$

where $\kappa$ was defined above. For small detuning $\kappa \ll$ $\left(-c_{0}\right)$, i.e. $1 / \tilde{v}_{0} \ll\left|\tilde{c}_{0}\right|^{3}$, a relation well-satisfied in the range in Fig 3 and others below, we then obtain $\tilde{D}_{0} \simeq$ $(32 \pi / 3)^{1 / 3}\left(-\tilde{c}_{0}\right)^{-1 / 2}$.

The "cross-over" behavior in Fig 3 is analogous to the $s$-wave case, where the corresponding $x$-axis is $x=$ $-1 /\left(n^{1 / 3} a_{s}\right)$ where $a$ is the $s$-wave scattering length. Note here $D_{0}$ has the dimension of $[\mathrm{E}][\mathrm{L}]^{-1}$ and behaves differently from the $s$-wave $\Delta$ in the BEC limit: it saturates rather than continue to increase. Rigorously speaking, we actually expect a (quantum) phase transition at the point where $\mu$ crosses zero [7, 22]: the system is gapless for $\mu>0$ but gapful if $\mu<0$. However, we found no evident changes of slope in $\mu$ and $\Delta$ when this transition is crossed, and thus expect this phase transition is very weak thermodynamically. Similar results were found in Ref 24] for the axial $\left(D_{1} \neq 0, D_{0}=D_{-1}=0\right.$ state).
We have also performed calculations for other values of $\tilde{c}_{0}$. The size of the crossover region is roughly proportional to the value of $\tilde{c}_{0}$ (which is in turn inversely proportional to the density). For example, for $\tilde{c}_{0}=-200$ (not shown), the corresponding results can be captured well by replacing the $x$-axis by $-1 / 2 \tilde{v}_{0}$ and dividing $\tilde{D}_{0}$ by $1 / \sqrt{2}$ in Fig 3

The above behavior applies only to sufficiently large $-\tilde{v}_{ \pm 1}^{-1}-\left(-\tilde{v}_{0}^{-1}\right)>0$. When this difference is sufficiently small, $\tilde{D}_{ \pm 1}$ will become finite. Similar to the treatment in Section II for the anisotropic weak-coupling BCS model, we first identify the critical $\tilde{v}_{ \pm 1}^{*-1}$ such that $D_{1}$ and/or $D_{-1}$ first start to deviate from zero. For simplicity, in the results below we shall take $c_{0}=c_{ \pm 1}$. This is satisfied to a good approximation at least for ${ }^{40} \mathrm{~K}$ near the resonances (see Fig 2). We linearize Eqs.(37)-(38) (and the latter with $m=1 \leftrightarrow m=-1)$ in $D_{1}$ and $D_{-1}$. We obtain

$$
-\frac{M}{4 \pi v_{1}^{*}}\left(\begin{array}{c}
D_{1} \\
D_{-1}^{*}
\end{array}\right)=\left(\begin{array}{cc}
A_{1} & A_{2} \\
A_{2} & A_{1}
\end{array}\right)\left(\begin{array}{c}
D_{1} \\
D_{-1}^{*}
\end{array}\right)
$$

where

$$
\begin{aligned}
A_{1}= & \frac{2 \pi}{L^{3}} \sum_{\vec{k}}\left|Y_{11}\right|^{2} h(\vec{k})-\frac{2 \pi}{L^{3}} \sum_{\vec{k}} D_{0}^{2}\left|Y_{10}\right|^{2}\left|Y_{11}\right|^{2} g(\vec{k}) \\
& -\frac{M^{2} c_{1}}{2 \pi \hbar^{2}}\left(\mu-\frac{3 M}{10 \pi \hbar^{2}} D_{0}^{2}\right) \\
A_{2}= & \frac{2 \pi}{L^{3}} \sum_{\vec{k}} D_{0}^{2}\left|Y_{10}\right|^{2}\left|Y_{11}\right|^{2} g(\vec{k})-\frac{3 M^{3} c_{1}}{40 \pi^{2} \hbar^{4}} D_{0}^{2} \\
g(\vec{k}) \equiv & \frac{k^{4}}{2}\left(\frac{1}{\left[\left(\epsilon_{k}-\mu\right)^{2}+D_{0}^{2}\left|Y_{10}\right|^{2} k_{z}^{2}\right]^{3 / 2}}-\frac{1}{\epsilon_{k}^{3}}\right)
\end{aligned}
$$

Here $h(\vec{k})$ is as defined in Eq. (36) but with only $D_{0} \neq$ 0 . We can verify that $A_{2}>0$. Therefore the largest eigenvalue for the A-matrix, hence the smallest value for $v_{1}^{*}$, belongs to the class $D_{1}=D_{-1}^{*}$. The corresponding critical value is then given by $-\frac{M}{4 \pi v_{1}^{*}}=A_{1}+A_{2}$. On the other hand, from Eq.(37), we have

$$
-\frac{M}{4 \pi v_{0}}=\frac{2 \pi}{L^{3}} \sum_{\vec{k}}\left|Y_{10}\right|^{2} h(\vec{k})-\frac{M^{2} c_{0}}{2 \pi \hbar^{2}}\left(\mu-\frac{9 M}{20 \pi \hbar^{2}} D_{0}^{2}\right)
$$

Combining these two equations and re-writing it in dimensionless form, we have

$$
\begin{aligned}
& -\frac{1}{\tilde{v}_{1}^{*}}+\frac{1}{\tilde{v}_{0}}=\frac{3\left(6 \pi^{2}\right)^{\frac{2}{3}}}{5 \pi} \tilde{D}_{0}^{2}\left(-\tilde{c}_{0}\right) \\
& +9 \pi \int_{0}^{\infty} d x \int_{-1}^{1} d y \frac{x^{4}\left(1-3 y^{2}\right)}{\left[\left(x^{2}-\tilde{\mu}\right)^{2}+\frac{3}{\pi} \tilde{D}_{0}^{2} x^{2} y^{2}\right]^{1 / 2}}
\end{aligned}
$$

In the BCS limit, $\tilde{D}_{0} \rightarrow 0$, the first term in the R.H.S. of Eq.(49) is negligible whereas the second, which we shall 
call $K_{1}$, is finite. The dominant contribution to $K_{1}$ occurs near $x \simeq \sqrt{\tilde{\mu}}$, where the integrand diverges if $\tilde{D}_{0}$ were zero exactly. To evaluate $K_{1}$ in the limit $\tilde{D}_{0} \rightarrow 0$, we add and subtract the integral

$$
K_{2} \equiv 9 \pi \int_{-\xi_{0}}^{\xi_{0}} d \xi \int_{-1}^{1} d y \frac{\left(1-3 y^{2}\right)}{2\left[\xi^{2}+\frac{3}{\pi} \tilde{D}_{0}^{2} y^{2}\right]^{\frac{1}{2}}}
$$

where $\xi \equiv x^{2}-\tilde{\mu}$, and $\xi_{0} \gg \tilde{D}_{0}$ is an arbitrary cut-off. The integrand for $K_{1}-K_{2}$ now has no divergence near $x^{2}-\tilde{\mu} \approx 0$, and their difference vanishes in the $\tilde{D}_{0} \rightarrow 0$ limit due to the $y$ integral. $K_{2}$ can be evaluated by first integrating with respect to $\xi$, we get

$$
\begin{aligned}
& 9 \pi \int_{-1}^{1} d y \int_{-\xi_{0}}^{\xi_{0}} d \xi \frac{\left(1-3 y^{2}\right)}{2\left[\xi^{2}+\frac{3}{\pi} \tilde{D}_{0}^{2} y^{2}\right]^{\frac{1}{2}}} \\
= & -18 \pi \int_{-1}^{1} d y\left(1-3 y^{2}\right) \log y \\
= & 12 \pi
\end{aligned}
$$

In going from the first to the second line, we have used the fact that $\int_{-1}^{1} d y\left(1-3 y^{2}\right)=0$, hence the terms involving $\xi_{0}$ and $\tilde{D}_{0}$ drop out in the $\xi_{0} \gg \tilde{D}_{0}$ limit. Therefore in the BCS limit we obtain $-\tilde{v}_{1}^{*-1}+\tilde{v}_{0}^{-1} \rightarrow 12 \pi=37.7$.

In the BEC limit, recalling that $\tilde{D}_{0}$ approaches a constant $\simeq(32 \pi / 3)^{1 / 3}\left(-\tilde{c}_{0}\right)^{-1 / 2}$ whereas $\tilde{\mu}$ is large and negative, we find that the second term in the RHS of Eq. (49) is negligible compared with the first (provided $\left.\kappa \ll\left(-c_{0}\right)\right)$. Using the value for $\tilde{D}_{0}$, Eq. (49) yields $-\tilde{v}_{1}^{*-1}+\tilde{v}_{0}^{-1} \rightarrow 48 \pi / 5=30.2$. In the intermediate regime, the numerical results for $-\tilde{v}_{1}^{*-1}+\tilde{v}_{0}^{-1}$ as a function of $-\tilde{v}_{0}^{-1}$ is shown as the thick black line $\left(\tilde{D}_{0}=0\right)$ in Fig 4

We now solve for the order parameters $D_{m}$ when $-\tilde{v}_{1}^{-1}+\tilde{v}_{0}^{-1}$ is less than the critical value. As already mentioned, we have already made use of gauge invariance to choose $D_{0}$ to be real. Under this choice, the solutions we found belong to the class $D_{1}=D_{-1}^{*}$. Writing $D_{1}=\left|D_{1}\right| e^{i \chi}, \Delta_{\vec{k}}$ then has the angular dependence $\propto D_{0} \hat{k}_{z}+i \sqrt{2}\left|D_{1}\right| \hat{k} \cdot \hat{a} \propto\left(\hat{k}_{z}-i \beta \hat{k} \cdot \hat{a}\right)$ where $\hat{a}=(\cos \chi) \hat{y}+(\sin \chi) \hat{x}$ is a unit vector perpendicular to $\hat{z}$ and $\beta=\sqrt{2}\left|D_{1} / D_{0}\right|$. A particular solution is given by the case where $D_{1}$ and $D_{-1}$ are both real where $\hat{a}=\hat{y}$. Other solutions are simply related to this one by a rotation about $\hat{z}$. Without loss of generality, we shall therefore assume that $D_{m}$ 's are all real below.

The contour plot of $\beta$ (by solving Eq. (37)-(40) without the $1 /\left|\alpha_{m}\right|^{2}$ terms) is shown in Fig 4 (see Fig. 2 of 28] for a plot of $\left.D_{ \pm 1}\right) \beta$ monotonically increases downwards or towards the right in this diagram. In the $\beta=0$ $\left(D_{ \pm 1}=0\right)$ phase, the state is rotationally invariant about $\hat{z}$, whereas this symmetry is broken in the $\beta \neq 0$ phase. There is a (quantum) phase transition between these two phases when one crosses the critical line $-\tilde{v}_{1}^{*-1}+\tilde{v}_{0}^{-1}$.

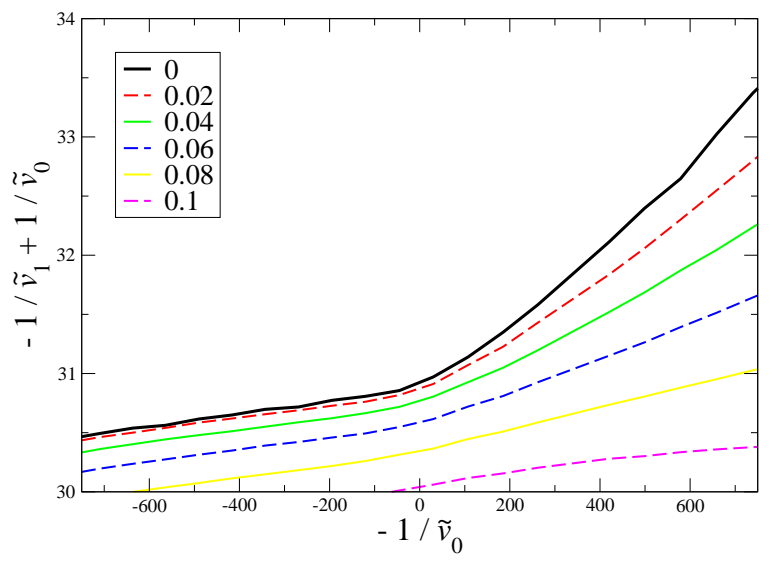

FIG. 4: Contour plot of $\beta$ as a function of $-1 / \tilde{v}_{1}+1 / \tilde{v}_{0}$ and $-1 / \tilde{v}_{0}$ for $\tilde{c}_{0}=\tilde{c}_{ \pm 1}=-100$. The line for $\beta=0$ corresponds to the critical value $-1 / \tilde{v}_{1}^{*}+1 / \tilde{v}_{0}$.

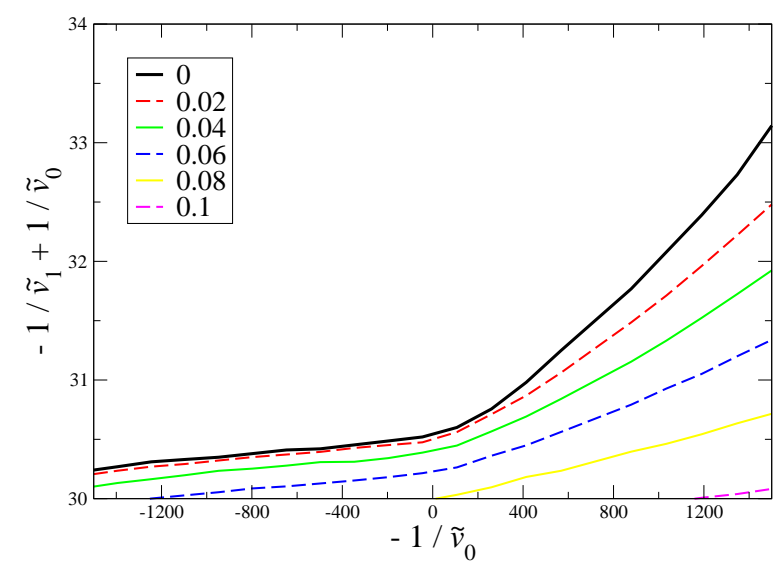

FIG. 5: Contour plot of $\beta$ as a function of $-1 / \tilde{v}_{1}+1 / \tilde{v}_{0}$ and $-1 / \tilde{v}_{0}$ for $\tilde{c}_{0}=\tilde{c}_{ \pm 1}=-200$.

For $\tilde{c}_{0}=-200$, the results are qualitatively the same provided we double the value of $-1 / \tilde{v}_{0}$. The corresponding plot is shown in Fig

In the intermediate splitting regime, we thus predict the state is $k_{z}-i \beta k_{y}$ on the BCS side, whereas it is $k_{z}$ on the BEC side. For large positive detuning, the splitting should be less relevant and the pairing state should resemble more that of the isotropic system. On the BEC side, the system should be closer to a Bose condensate of lowest energy molecules $\left(\hat{k}_{z}\right)$. 
For the ${ }^{40} \mathrm{~K}$ case studied in Ref. [19], the Feshbach resonances are at $B_{0}^{*} \approx 198.8 \mathrm{G}$ and $B_{1}^{*} \approx 198.4 \mathrm{G}$. There, $c_{1}$ and $c_{0}$ are both only weakly field dependent and are approximately given by $-0.02 a_{0}^{-1}$ (see Fig 2). Our choice of $\tilde{c}=-100$ above corresponds to a density of roughly $10^{-11} a_{0}^{-3}=6.7 \times 10^{13} \mathrm{~cm}^{-3}$. The range of $-1 / \tilde{v}_{0}$ for $\tilde{\mu}$ to go from 0 to 1 corresponds to roughly $0.5 \mathrm{G}$ if we take the same gas density. (This field range is proportional to $n^{2 / 3}$ ). Near the resonant fields, $-v_{1}^{-1}+v_{0}^{-1} \approx 2.1 \times 10^{-8} a_{0}^{-3}$ and is roughly field independent. Thus the density determines the values for both $\tilde{c}_{0,1}$ and $-\tilde{v}_{1}^{-1}+\tilde{v}_{0}^{-1}$ while varying the magnetic field corresponds roughly to moving along a horizontal line on our phase diagram of Fig 4 (with increasing field towards the right and the distance of the line from the horizontal axis proportional to $n^{-1}$ ). For the density cited above, $-\tilde{v}_{1}^{-1}+\tilde{v}_{0}^{-1} \approx 2000$, hence we expect only the $k_{z}$ phase to be observed. To observe the phase transition, we need $\mathrm{a}^{40} \mathrm{~K}$ gas of a much higher density, or another gas species with much smaller splitting between the $m=0$ and $m= \pm 1$ resonances.
In the above treatment, we have assumed that the terms with explicit $1 /\left|\alpha_{m}\right|^{2}$ factors in Eqs.(37)-(40) can be dropped. The validity of this assumption has to be investigated by first-principle calculations of two-atom collisions. Following Ref [37], we define the quantity

$$
\eta_{m} \equiv \frac{M^{2}\left|\alpha_{m}\right|^{2}}{\hbar^{4} L^{3}} \sum_{\vec{p}} \frac{1}{p^{2}} .
$$

$\eta_{m}$ quantifies the relative strength of coupling between the $m$ closed-channel and the continuum. With this notation, Eq. (34) becomes

$$
c_{m}=-\frac{4 \pi \hbar^{4}}{M^{2}\left|\alpha_{m}\right|^{2}}\left(1+\eta_{m}\right) .
$$

The single channel approximation used above corresponds to $\eta_{m} \rightarrow \infty$. We now investigate the effect of finite $\eta_{m}$ on the many-body problem. Expressing the Eqs.(37), (38), and (40) by $\eta_{m}$ instead of $1 /\left|\alpha_{m}\right|^{2}$, we get

$$
\begin{array}{r}
\frac{M}{4 \pi} D_{0}\left(-\frac{1}{v_{0}}+\frac{2 M c_{0} \mu}{\hbar^{2}}\right)-\frac{\eta_{0}}{1+\eta_{0}} \frac{3 M^{3} c_{0}}{40 \pi^{2} \hbar^{4}}\left[D_{0}\left(2\left|D_{1}\right|^{2}+3\left|D_{0}\right|^{2}+2\left|D_{-1}\right|^{2}\right)-2 D_{0}^{*} D_{1} D_{-1}\right] \\
=\frac{2 \pi}{L^{3}} \sum_{\vec{k}, m} D_{m} Y_{10}^{*}(\hat{k}) Y_{1 m}(\hat{k}) h(\vec{k}) \\
\frac{M}{4 \pi} D_{1}\left(-\frac{1}{v_{1}}+\frac{2 M c_{1} \mu}{\hbar^{2}}\right)-\frac{\eta_{1}}{1+\eta_{1}} \frac{3 M^{3} c_{1}}{40 \pi^{2} \hbar^{4}}\left[D_{1}\left(3\left|D_{1}\right|^{2}+2\left|D_{0}\right|^{2}+6\left|D_{-1}\right|^{2}\right)-D_{-1}^{*} D_{0}^{2}\right] \\
=\frac{2 \pi}{L^{3}} \sum_{\vec{k}, m} D_{m} Y_{11}^{*}(\hat{k}) Y_{1 m}(\hat{k}) h(\vec{k})
\end{array}
$$

$$
\begin{aligned}
n= & \frac{1}{2 L^{3}} \sum_{\vec{k}}\left(1-\frac{\epsilon_{k}-\mu}{\left[\left(\epsilon_{k}-\mu\right)^{2}+\left|\Delta_{\vec{k}}\right|^{2}\right]^{\frac{1}{2}}}-\frac{\left|\Delta_{\vec{k}}\right|^{2}}{2 \epsilon_{k}^{2}}\right) \\
& -\frac{M^{2}}{(4 \pi)^{2} \hbar^{4}} \sum_{m} \frac{2+\eta_{m}}{1+\eta_{m}}\left|D_{m}\right|^{2} c_{m}
\end{aligned}
$$

We note here that the last term of Eq.(156) involves a factor $\left(2+\eta_{m}\right) /\left(1+\eta_{m}\right)$. This term actually arises from two contributions. The first one, involving $2 /\left(1+\eta_{m}\right)$, is due to the closed-channel molecules (the last term in Eq.(40) ), and a second one, involving $\eta_{m} /\left(1+\eta_{m}\right)$ due to the open-channel atoms.

To show the effect of finite $\eta_{0}$, we plot the general results for $\tilde{D}_{0}$ and $\tilde{\mu}$ in the state $\tilde{D}_{ \pm 1}=0$ in Fig 6
The value of $\eta_{0}$ mainly affects the results on the BEC side. In fact, deep in the BCS limit the results are independent of $\eta_{0}$, as expected since all atoms are basically in the open-channels. $\tilde{\mu}$ is still basically linear in $1 / \tilde{v}_{0}$ with the same slope, and thus the width of the "crossover" region remains to be determined by $\tilde{c}_{0}$ only (but not $\eta_{0}$ ). In the BEC limit, when $\eta_{0}$ decreases from $\infty$, the contribution from bound state molecules becomes more important (see in particular Eq. (56) and the discussions below it). The chemical potential $\tilde{\mu}$ becomes closer to the two-body value for decreasing $\eta_{0}$ as shown in Fig. 6(b). $\tilde{D}_{0}$ has the limiting value $\simeq(32 \pi / 3)^{1 / 3}\left(-\tilde{c}_{0}\right)^{-1 / 2}\left[\left(1+\eta_{0}\right) /\left(2+\eta_{0}\right)\right]^{1 / 2}$, and thus decreases with decreasing $\eta_{0}$.

The phase transition between the $k_{z}$ state and the $k_{z}-$ $i \beta k_{y}$ state can be investigated as before. The value for $-\tilde{v}_{1}^{*-1}+\tilde{v}_{0}^{-1}$ is not affected by the value of $\eta_{m}$ 's in the BCS limit. In the BEC limit, if $\eta_{0}=\eta_{ \pm 1} \equiv \eta$, we obtain 


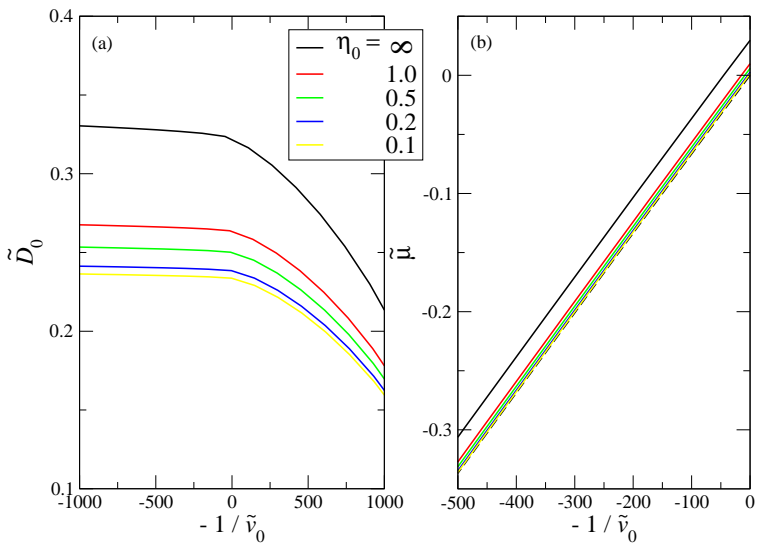

FIG. 6: Plots of (a) $\tilde{D}_{0}$ and (b) $\tilde{\mu}$ as functions of $-1 / \tilde{v}_{0}$ at $\tilde{c}_{0}=\tilde{c}_{ \pm 1}=-100, \tilde{D}_{ \pm 1}=0$ for different $\eta_{0}$ 's. $\eta_{0}=\infty$ corresponds that of Fig 3 The dashed line in (b) represents to the two-body result.

$-\tilde{v}_{1}^{*-1}+\tilde{v}_{0}^{-1} \rightarrow(30.2) \eta /(2+\eta)$. This critical value thus decreases with decreasing $\eta$. Its behavior is as shown in Fig 7 The $\eta$ dependence of this transition line has also been investigated in Ref [29].

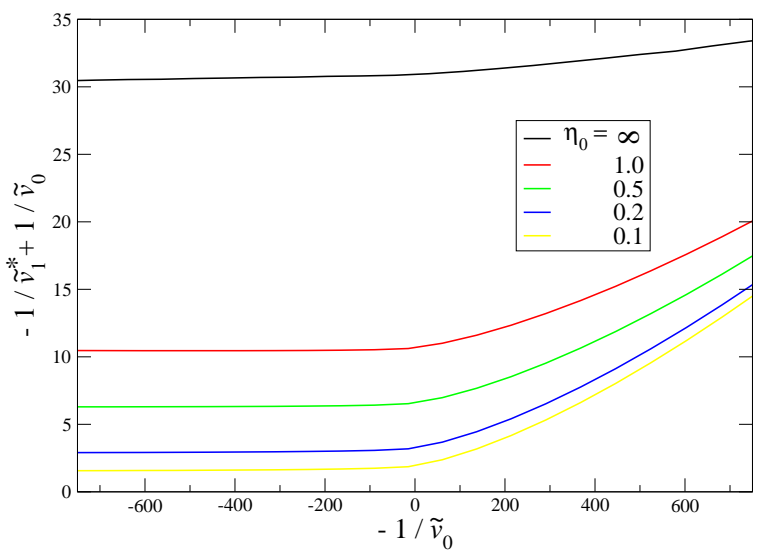

FIG. 7: The transition line $-\tilde{v}_{1}^{*-1}+\tilde{v}_{0}^{-1}$ between the $k_{z}$ phase and the $k_{z}-i \beta k_{y}$ phase for $\eta$ 's in the legend. $\tilde{c}_{0}=\tilde{c}_{ \pm 1}=$ -100 .

We conclude with a discussion on the condition $\eta \gg 1$, or equivalently $\hbar^{4} /\left(\alpha_{m} M\right)^{2} \ll\left|c_{m}\right|$, for the validity of the effective single-channel approximation. It is worth noting that the corresponding condition for the s-wave Feshbach resonance superfluidity is quite different. For this latter case, the single-channel approximation near resonanace is valid if $32,33,34,35,36]$ the corresponding parame- ter $\alpha_{s}$ in Eq.(19) satisfies $\hbar^{4} /\left(\left|\alpha_{s}\right|^{2} M^{2}\right) \ll 1 / n^{1 / 3}$. This case is often also referred to the "wide resonance" regime. Though for both $\mathrm{s}$ and $\mathrm{p}$-wave resonances, the singlechannel approximation would be valid for sufficiently strong coupling (large $\alpha$ ) between the closed and open channels, for s-wave the condition for a single-channel approximation is always satisfied when the gas is sufficiently dilute. In contrast, for p-wave the condition $\eta \gg 1$ is independent of the density of the gas. This difference can be understood by noting that the dimension of $\alpha_{s}$ is $[\mathrm{E}][\mathrm{L}]^{3 / 2}$, whereas that of $\alpha_{m}$ here is $[\mathrm{E}][\mathrm{L}]^{5 / 2}$. The effective range $r_{s}$ for s-wave Feshbach resonance [32, 33] is proportional to $\hbar^{4} /\left(\left|\alpha_{s}\right|^{2} M^{2}\right)$, and the additional term $\propto \sum\left(1 / p^{2}\right)$ in Eq. (34) does not arise.

For the s-wave case, the terms "single-channel approximation" and "wide-resonance" have been used interchangably. However, we should note that for our p-wave case, the condition $\eta \gg 1$ is not necessarily in conflict with the fact that the the peak in scattering cross-section discussed in Fig.1 of Ref. [19] is narrow. This p-wave scattering cross-section is proportional to $\left|f_{m}\right|^{2}$, which is given by

$$
\left|f_{m}\right|^{2}=\frac{k^{4}}{\left(\frac{1}{v_{m}}+\left|c_{m}\right| k^{2}\right)^{2}+k^{6}}
$$

For $B>B_{m}^{*}$, where $1 / v_{m}<0$, the cross-section thus has a peak at finite energy $E=\hbar^{2} k_{o}^{2} / M$ where $k_{o}^{2}=1 /\left(\left|c_{m} v_{m}\right|\right)$. The denominator can be written as $c_{m}^{2}\left(k^{2}-k_{o}^{2}\right)^{2}+k^{6}$. For $B \gtrsim B_{m}^{*},\left|f_{m}\right|^{2}$ as a function of $k^{2}$ then peaks very near $k^{2}=k_{o}^{2}$ and has a width approximately given by $k_{o}^{3} /\left|c_{m}\right|=1 /\left(\left|c_{m}\right|^{5 / 2}\left(-v_{m}\right)^{3 / 2}\right) \ll k_{o}^{2}$, and therefore a very narrow (in energy) resonance. This is entirely a result of the special form of $f$ for finite angular momentum. This narrowness of the peak is therefore not directly connected with the assumption on the magitude of $\eta$ made here.

In addition, the condition for $\eta \gg 1$ here is also not necessarily in conflict with the assumption of a "narrow" resonance in the context discussed by Gurarie et al 29]. They defined a parameter $\gamma \propto \alpha_{m}^{2} M^{2} n^{1 / 3}$, and called the case $\gamma \ll 1$ to be a narrow resonance. Their condition is fulfilled always at sufficiently low densities. Thus the resonance can be narrow in their language whereas the single-channel approximation can still be valid if $n^{1 / 3} \ll$ $\hbar^{4} /\left(\alpha_{m} M\right)^{2} \ll\left|c_{m}\right|$.

\section{EXPERIMENTAL PROBE OF PAIRING SYMMETRY}

It was suggested by Altman et al. 38 that the measurement of the atom shot noise in the time-of-flight (TOF) absorption image can reveal the properties of the many-body states, including the symmetry of the pairing state. Later the proposal was carried out by Greiner 
et al. [39] for ${ }^{40} \mathrm{~K}$ atoms under an $s$-wave Feshbach resonance. As discussed in previous section, the pairing state induced by $p$-wave Feshbach resonance is either $k_{z}$ or $k_{z}-i \beta k_{y}$ where $\beta$ can vary under a change in the magnetic field or the gas density. In this section, we propose how $\beta$ can be determined in a TOF experiment.

In the TOF experiment, one can measure the quantity

$$
\begin{aligned}
\mathcal{G}(\vec{r},-\vec{r}) & \equiv\langle n(\vec{r}) n(-\vec{r})\rangle_{t}-\langle n(\vec{r})\rangle_{t}\langle n(-\vec{r})\rangle_{t} \\
& \propto\left\langle n_{\vec{k}} n_{-\vec{k}}\right\rangle-\left\langle n_{\vec{k}}\right\rangle\left\langle n_{-\vec{k}}\right\rangle
\end{aligned}
$$

where $\vec{k} \equiv m \vec{r} /(\hbar t) . \quad t$ is the time after the trapping potential and the interaction between atoms are suddenly turned off. Using the mean-field Hamiltonian in Eq.(22), we have

$$
\begin{aligned}
\mathcal{G}(\vec{r},-\vec{r}) & \propto \frac{\left|\Delta_{\vec{k}}\right|^{2}}{\left(\epsilon_{k}-\mu\right)^{2}+\left|\Delta_{\vec{k}}\right|^{2}} \\
& =\frac{\frac{3}{\pi} \tilde{D}_{0}^{2}\left(\tilde{k}_{z}^{2}+\beta^{2} \tilde{k}_{x}^{2}\right)}{\left(\tilde{k}^{2}-\tilde{\mu}\right)^{2}+\frac{3}{\pi} \tilde{D}_{0}^{2}\left(\tilde{k}_{z}^{2}+\beta^{2} \tilde{k}_{x}^{2}\right)}
\end{aligned}
$$

where $\tilde{\vec{k}} \equiv \vec{k} / k_{\mathrm{F}}$. The two phases $k_{z}$ and $k_{z}-i \beta(\vec{k} \cdot \hat{a})$ can be distinguished by the presence of anisotropy in the $x-y$ plane. For example, consider the weighted average over $\phi$ defined by

$$
S_{0}(\alpha) \equiv\langle\cos (2(\phi+\alpha)) \mathcal{G}(\vec{r},-\vec{r})\rangle
$$

This average vanished in the $k_{z}$ phase. For $\beta \neq 0, S_{0}(\alpha)$ becomes finite and can in principle be evaluated. The presence of the $\tilde{k}_{z}^{2}+\beta^{2} \tilde{k}_{x}^{2}$ term in the denominator complicates the analysis. There are nevertheless two possible simplifications. In the BEC limit, the terms involving $\tilde{D}_{0}^{2}$ in the denominator are negligible compared with the first. In the BCS limit we can confine ourselves to data where $r \gg R_{\mathrm{F}}$, equivalently $k \gg k_{\mathrm{F}}$. In both cases the correlation function has the angular dependence

$$
\mathcal{G}(\vec{r},-\vec{r}) \propto\left(\tilde{k}_{z}^{2}+\beta^{2} \tilde{k}_{x}^{2}\right)
$$

Then, for $\hat{a}=(\cos \chi) \hat{y}+(\sin \chi) \hat{x}$ as before, we get

$$
S_{0}(\alpha) \propto-D_{0}^{2} \beta^{2} \cos (2(\chi-\alpha)) .
$$

Thus $S_{0}(\alpha)$ will reveal the finiteness of $\beta$ as well as the value of $\chi$ hence the direction of $\hat{a}$ (up to a sign). The values of $\beta$ however are still difficult to determine in this way, both because the need to evaluate the proportionality coefficient in $S_{0}$ as well as the fact that experiments so far detect a correlation in s-wave substantially less than the expected theoretical value. However, we can consider instead

$$
\begin{aligned}
& S_{1} \equiv\left\langle\cos ^{2}(\hat{r} \cdot \hat{z}) \mathcal{G}(\vec{r},-\vec{r})\right\rangle \\
& S_{2} \equiv\left\langle\sin ^{2}(\hat{r} \cdot \hat{z}) \mathcal{G}(\vec{r},-\vec{r})\right\rangle
\end{aligned}
$$

These give

$$
\frac{S_{1}}{S_{2}}=\frac{3+\beta^{2}}{2+4 \beta^{2}}
$$

and hence the value of $\beta$ can be determined via

$$
\beta^{2}=\frac{3-2\left(S_{1} / S_{2}\right)}{4\left(S_{1} / S_{2}\right)-1}
$$

For the polar state, $\beta=0$ and $S_{1}=3 S_{2} / 2$. For the axial state, $\beta=1$ with $S_{1}=2 S_{2} / 3$.

\section{CONCLUSION}

In conclusion, due to the magnetic dipolar interaction between two fermionic atoms, the superfluidity in trapped gases induced by a $p$-wave Feshbach resonance is expected to behave very differently from the more familiar example of ${ }^{3} \mathrm{He}$. The symmetry of the ground state can be tuned by varying either the density of the gas or the applied external magnetic field. The phase can be a polar state or a state intermediate between the polar and the axial phase. We also propose how the pairing symmetry can be detected from an experimental investigation.

\section{ACKNOWLEDGEMENT}

S.K.Y. is very grateful to T.-L. Ho and A. J. Leggett for useful discussions. This research is supported by the National Science Foundation under Grant No. PHY9907949 and the National Science Council of Taiwan under Grant Nos. NSC93-2112-M-001-016 and NSC94-2112-M001-002. The postdoctoral position of C.H.C. is supported by NSC under Grant No. NSC93-2816-M-0010007-6 and Academia Sinica.

[1] F. Dalfovo et al., Rev. Mod. Phys. 71, 463 (1999) and references therein.

[2] Y. Castin, in Coherent atomic matter waves, Les Houches Session LXXII, NATO Advanced Study Institute, edited by R. Kaiser, C. Westbrook, and F. David (EDP Science; Spinger-Verlag, 2001) and references therein.

[3] W. C. Stwalley, Phys. Rev. Lett. 37, 1628 (1976)

[4] E. Tiesinga et al., Phys. Rev. A 46, R1167 (1992); ibid, Phys. Rev. A 47, 4114 (1993)

[5] A.J. Leggett, J. Phys. (Paris), Colloq. 41, C7-19 (1980).

[6] P. Nozieres and S. Schmitt-Rink, J. Low Temp. Phys. 59, 195 (1985).

[7] M. Randeria, J.-M. Duan and L.-Y. Shieh, Phys. Rev. B 41, 327 (1990)

[8] Y. Ohashi and A. Griffin, Phys. Rev. A 67, 033603 (2003).

[9] G. M. Falco and H. T. C. Stoof, Phys. Rev. Lett. 92, 130401 (2004)

[10] A. Perali, Phys. Rev. Lett. 92, 220404 (2004); ibid, 93, 100404 (2004)

[11] J. Javanainen et al, Phys. Rev. Lett. 95, 110408 (2005) 
[12] S.-Y. Chang et al, Phys. Rev. A, 70, 043602 (2004); G. Astrakharchik et al Phys. Rev. Lett. 93, 200404 (2004); A. Bulgac et al cond-mat/0505374 (2005)

[13] C.A. Regal, Phys. Rev. Lett. 92, 040403 (2004).

[14] T. Bourdel et al., Phys. Rev. Lett. 93, 050401 (2004).

[15] M.W. Zwierlein et al., Phys. Rev. Lett. 92, 120403 (2004)

[16] M. Bartenstein et al., Phys. Rev. Lett. 92, 120401 (2004).

[17] J. Kinast et al., Science 307, 1296 (2005).

[18] C.A. Regal et al., Phys. Rev. Lett. 90, 053201 (2003).

[19] C. Ticknor et al., Phys. Rev. A 69, 042712 (2004).

[20] J. Zhang et al., Phys. Rev. A 70, 030702(R) (2004).

[21] K. Günter et al., cond-mat/0507632 (2005)

[22] F.R. Klinkhamer and G.E. Volovik, JETP Lett. 80, 389 (2004).

[23] Y. Ohashi, Phys. Rev. Lett. 94, 050403 (2005).

[24] T.L. Ho and R.B. Diener, Phys. Rev. Lett. 94, 090402 (2005).

[25] For an alternative mechanism for $\mathrm{p}$-wave in trapped Fermi gases, see L. You and M. Marinescu, Phys. Rev. A 60, 2324 (1999).

[26] A.J. Leggett, Rev. Mod. Phys. 75, 331 (1975).

[27] P.W. Anderson and P. Morel, Phys. Rev. 123, 1911
(1961).

[28] C.H. Cheng and S.-K. Yip, Phys. Rev. Lett. 95, 070404 (2005).

[29] V. Gurarie, L. Radzihovsky, and A.V. Andreev, Phys. Rev. Lett. 94, 230403 (2005); see also cond-mat/0410620 3 .

[30] E. Timmermanns et al., Phys. Rep. 315, 199 (1999); M. Holland et al, Phys. Rev. Lett. 87, 120406 (2001)

[31] E. Merzbacher, Quantum Mechanics, John Wiley and Sons, New York (1970)

[32] R. Combescot, Phys. Rev. Lett. 91, 120401 (2003); G.M. Bruun and C.J. Pethick, ibid 92, 140404 (2004).

[33] R.B. Diener and T.-L. Ho, cond-mat/0405174

[34] M. Mackie and J. Piilo, Phys. Rev. Lett. 94, 060403 (2005)

[35] M.H. Szymanska et al., cond-mat/0501728

[36] G. B. Partridge et al, Phys. Rev. Lett. 95, 020404 (2005)

[37] F. Chevy et al., Phys. Rev. A 71, 062710 (2005).

[38] E. Altman, E. Demler, and M.D. Lukin, Phys. Rev. A 70, 013603 (2004).

[39] M. Greiner, C.A. Regal, J.T. Stewart, and D.S. Jin, Phys. Rev. Lett. 94, 110401 (2005). 